\title{
Invariant preserving schemes for double dispersion equations
}

\author{
Natalia Kolkovska ${ }^{1 *}$ (D) and Veselina Vucheva ${ }^{1}$
}

${ }^{*}$ Correspondence: natali@math.bas.bg

${ }^{1}$ Institute of Mathematics and Informatics of the Bulgarian

Academy of Sciences, Sofia, Bulgaria

\begin{abstract}
We propose and study two finite difference schemes (FDSs) for the double dispersion equations. The first FDS is symplectic, while the second one preserves the discrete momentum exactly. Both FDS conserve the discrete energy approximately with $O\left(h^{2}+\tau^{2}\right)$ global error. The extensive numerical experiments agree well with the theoretical results for single solitary wave as well as for the interaction between two solitary waves.
\end{abstract}

PACS Codes: $62.30 .+\mathrm{d} ; 45.20 . \mathrm{Jj}$

Keywords: Double dispersion equation; Boussinesq equation; Symplectic scheme; Hamiltonian system; Mass, momentum and energy conservation schemes

\section{Introduction}

The aim of this paper is to develop and analyze two finite difference schemes (FDSs) for the solution of the double dispersion equations (DDEs)

$$
\frac{\partial^{2} u}{\partial t^{2}}=\Delta u+\beta_{1} \Delta \frac{\partial^{2} u}{\partial t^{2}}-\beta_{2} \Delta^{2} u+\Delta f(u), \quad x \in R, t>0,
$$

with initial data

$$
u(x, 0)=u_{0}(x), \quad \frac{\partial u}{\partial t}(x, 0)=u_{1}(x), \quad x \in R,
$$

and asymptotic boundary conditions $u(x, t) \rightarrow 0, \Delta u(x, t) \rightarrow 0$ as $|x| \rightarrow \infty$.

Here the dispersion parameters $\beta_{1}, \beta_{2}$ satisfy $\beta_{1} \geq 0, \beta_{2}>0$. The nonlinear term has the form $f(u)=\alpha u^{p}, p=2,3, \ldots$, with $\alpha$ an amplitude parameter.

The DDE appears in a number of mathematical models of physical processes, for example-in the modeling of surface waves in shallow water, in the dislocation theory of crystals, in optics, etc. The derivation of (1) from the full Boussinesq model can be found e.g. in [10], where Eq. (1) is also called the Boussinesq paradigm equation.

The problem (1) with $\beta_{1}=0$ is referred in the literature as Boussinesq equation. Several numerical methods are used to solve this equation. For example, a Galerkin method is investigated in [8, 23], a finite difference method is applied in [11], spectral and pseudospectral methods are reported in $[6,12,22,27]$. A predictor-corrector method is derived in [2], while a mesh-free method is given in [21]. Numerical analysis of the Boussinesq

(c) The Author(s) 2019. This article is distributed under the terms of the Creative Commons Attribution 4.0 International License (http://creativecommons.org/licenses/by/4.0/), which permits unrestricted use, distribution, and reproduction in any medium, provided you give appropriate credit to the original author(s) and the source, provide a link to the Creative Commons license, and indicate if changes were made. 
equation based on structure preserving methods (symplectic and multi-symplectic methods) is reported in $[1,3-5,15,26]$.

The DDE (1) with $\beta_{1}>0$ is studied less numerically. The finite difference method is used in $[7,9,10,16,18]$ to solve the one-dimensional problem (1). The exact preservation of the global discrete energy and the convergence of the numerical methods are proved in [17-19].

The DDE conserves not only the energy, it preserves the mass, the momentum; and it has a Hamiltonian structure-the phase flow of the Hamiltonian systems are symplectic transformations that conserve area.

We believe, that the numerical methods should preserve as much of the essential properties of the original continuous problem as possible. Based on this idea we construct in this paper two new invariant preserving schemes for DDE, called FDS-S and FDS-M. We define discrete invariants of the mass, the momentum and the energy (equivalently the Hamiltonian) and analyze conservation of these quantities in time. The FDS-S is symplectic as the continuous problem is, it retains at the discrete level the symplecticness of the flow. In addition we prove that this scheme satisfies equalities, which connect the discrete momentum on every two consecutive time levels; the same holds true for the discrete energy. Moreover, we show that FDS-S preserves the discrete momentum and the discrete Hamiltonian in time approximately, up to $O\left(h^{2}+\tau^{2}\right)$ global error.

We prove that the second FDS, i.e. FDS- $M$, preserves exactly the discrete momentum and approximately, up to $O\left(h^{2}+\tau^{2}\right)$ global error, the discrete Hamiltonian. In addition both FDS preserve exactly the discrete mass.

We report and discuss numerical experiments based on two examples-one solitary wave and interaction of two solitary waves. We study on nested grids the convergence of the discrete solution to the exact one, as well as the approximate preservation of the discrete momentum and the discrete energy. The provided numerical tests are in accordance with theoretical results.

The paper is organized in the following way. The necessary preliminary results are reported in Sect. 2. The two FDS are stated in Sect. 3. The theoretical analysis of these FDS is given in Sect. 4. In Sect. 5 we report and discuss numerical experiments on two examples. Our concluding remarks are given in Sect. 6.

\section{Preliminaries}

DDE (1) can be rewritten as the generalized Hamiltonian system (or Poisson system)

$$
\left[\begin{array}{l}
\frac{\partial u}{\partial t} \\
\frac{\partial v}{\partial t}
\end{array}\right]=J\left[\begin{array}{l}
\frac{\delta H}{\delta u} \\
\frac{\delta H}{\delta v}
\end{array}\right]
$$

using auxiliary function $v$, defined by $\frac{\partial v}{\partial x}=\frac{\partial u}{\partial t}$. Here

$$
J=\left[\begin{array}{cc}
0 & \left(E-\beta_{1} \Delta\right)^{-1} \partial_{x} \\
\left(E-\beta_{1} \Delta\right)^{-1} \partial_{x} & 0
\end{array}\right]
$$

is an anti-symmetric matrix and $E$ is the identity operator. The separable Hamiltonian

$$
H(t):=H(u(x, t), v(x, t))=\frac{1}{2} \int_{\mathbb{R}}\left(v^{2}+\beta_{1}\left(\frac{\partial v}{\partial x}\right)^{2}+u^{2}+\beta_{2}\left(\frac{\partial u}{\partial x}\right)^{2}+2 F(u)\right) d x
$$


represents the total energy of the system, $F(u)=\int_{0}^{u} f(s) d s$ and $\frac{\delta H}{\delta u}, \frac{\delta H}{\delta v}$ are the variational derivatives of $H$ with respect to $u$ and $v$. The Poisson bracket is defined in this case as $\omega\left(\mathbf{G}_{\mathbf{1}}, \mathbf{G}_{\mathbf{2}}\right)=\nabla\left(\mathbf{G}_{\mathbf{1}}\right)^{T} J \nabla\left(\mathbf{G}_{\mathbf{2}}\right)$; see e.g. [14, 20].

The scalar DDE (1)-(2) is equivalent to the system

$$
\left\{\begin{array}{l}
\frac{\partial u}{\partial t}=\frac{\partial v}{\partial x}, \\
\frac{\partial v}{\partial t}=\left(E-\beta_{1} \Delta\right)^{-1}\left(\frac{\partial u}{\partial x}-\beta_{2} \frac{\partial^{3} u}{\partial x^{3}}+\frac{\partial f(u)}{\partial x}\right),
\end{array}\right.
$$

with initial conditions

$$
u(x, 0)=u_{0}(x), \quad v(x, 0)=v_{0}(x), \quad \text { where } \partial_{x} v_{0}(x)=u_{1}(x) .
$$

Given a solution $(u, v)$ of $(4)$ we define the mass $I(u(x, t))$ and the momentum $M(u(x, t)$, $v(x, t))$ as

$$
\begin{aligned}
& I(t):=I(u(x, t))=\int_{\mathbb{R}} u(x, t) d x, \\
& M(t):=M(u(x, t), v(x, t))=\int_{\mathbb{R}}\left(u(x, t) v(x, t)+\beta_{1} u_{x}(x, t) v_{x}(x, t)\right) d x .
\end{aligned}
$$

The phase flow of the Hamiltonian system (4) is a symplectic transformation that preserves area (see [14]). Besides the symplectic property of (4), it has three invariants-the mass, the momentum, and the energy (which coincides with the Hamiltonian in this paper). In the following theorem we give an exact formulation of this statement.

Theorem 1 (Conservation laws) For every $t \geq 0$ the solution $(u, v)$ to the problem (4) satisfies the following identities:

$$
\begin{aligned}
& I(t)=I(0), \quad \text { i.e. mass conservation; } \\
& M(t)=M(0), \quad \text { i.e. momentum conservation; } \\
& H(t)=H(0), \quad \text { i.e. energy (Hamiltonian) conservation. }
\end{aligned}
$$

It is well known that the DDE possesses exact solutions of the form of traveling waves $u(x, t)=\psi(x-c t)$, where $c$ is the velocity of the wave. When these solutions are localized in space, maintain their shape during the evolution, interact with other solutions and emerge from the collision unchanged (except for a phase shift), then these solutions are called 'solitary waves' or 'solitons'. In the case of quadratic nonlinearity $\left(f(u)=\alpha u^{2}\right)$ the solitary waves to (1) are given by

$$
\varphi(x, t ; c)=\frac{3\left(c^{2}-1\right)}{2 \alpha} \operatorname{sech}^{2}\left(-\frac{1}{2} \sqrt{\frac{c^{2}-1}{\beta_{1} c^{2}-\beta_{2}}}(x-c t)\right) .
$$

Note that the pair $(\varphi(x, t ; c),-c \varphi(x, t ; c))$ is the solitary wave solution to the system (4).

In the following we suppose that $\beta_{1}>0$ and that the unique exact solution to (1)-(2) exists and is sufficiently smooth in the considered time interval $t \in[0, T]$. The paper [25] 
gives conditions on the initial data, under which the solution to (1)-(2) is sufficiently smooth.

By $C$ (with different indices) we denote positive numbers which depend on some norms of the functions $u$ and $v$ but are independent of the discretization steps $h$ and $\tau$.

\section{The finite difference schemes}

\subsection{Mesh and notations}

Theoretical analysis of the solitary waves (7) shows that waves decay exponentially to zero as $|x| \rightarrow \infty$. In view of this and the imposed asymptotic boundary conditions we choose numbers $L_{1}$ and $L_{2}$ sufficiently large, so that the exact solution with its derivatives is negligible outside the interval $\left[-L_{1}, L_{2}\right]$.

For simplicity of the numerical analysis presented in this paper we suppose that the discrete solution to DDE satisfies periodic boundary conditions at $-L_{1}$ and $L_{2}$.

In $\left[-L_{1}, L_{2}\right]$ we introduce an uniform grid $x_{i}, i=0,1, \ldots, N$ with step $h=\left(L_{1}+L_{2}\right) / N$. We denote the approximation of the function $u\left(x_{i}, t\right)$ at the mesh points $x_{i}$ by $U_{i}(t)$ and the approximations of $v\left(x_{i}, t\right)$ by $V_{i}(t)$. We use the following notations: $\mathbf{U}(\mathbf{t})=$ $\left(U_{1}(t), U_{2}(t), \ldots, U_{N}(t)\right), \mathbf{V}(\mathbf{t})=\left(V_{1}(t), V_{2}(t), \ldots, V_{N}(t)\right)$,

$$
\begin{aligned}
& \partial_{\hat{x}} U_{i}=U_{\hat{x}, i}=\frac{U_{i+1}-U_{i-1}}{2 h}, \quad \hat{\Delta}_{h} U_{i}=U_{\hat{x} \hat{x}, i}=\frac{U_{i+2}-2 U_{i}+U_{i-2}}{4 h^{2}}, \\
& U_{\hat{x} \hat{x}, i}=\frac{U_{i+3}-3 U_{i+1}+3 U_{i-1}-U_{i-3}}{8 h^{3}} .
\end{aligned}
$$

Denote by $\langle V, W\rangle=\sum_{i=1}^{N} h V_{i} W_{i}$ the discrete scalar product of the functions $V$ and $W$. For periodic functions $\left(U_{i}=U_{N+i}\right)$ the discrete operator $A U_{i}=-\hat{\Delta}_{h} U_{i}, i=1,2, \ldots, N$ is self-adjoint and positive definite, thus it has an inverse $A^{-1}$.

For the discretization in time we consider an uniform mesh $\left\{t^{k}=k \tau, k=0, \ldots, K, K=\right.$ $T / \tau$ \} with time step $\tau$ and discrete time differences

$$
U_{t}^{k}=\frac{U^{k+1}-U^{k}}{\tau}, \quad U_{\bar{t}}^{k}=\frac{U^{k}-U^{k-1}}{\tau} .
$$

The discrete approximations to $u\left(x_{i}, t^{k}\right)$ and $v\left(x_{i}, t^{k}\right)$ are denoted by $U_{i}^{k}$ and $V_{i}^{k}$, respectively. We omit the notation ${ }_{i}^{k}$ for the arguments of the mesh functions whenever possible.

\subsection{Finite difference scheme FDS-S}

Replacing the derivatives in (4) with finite differences of second order of approximation, we obtain the semi-discrete system of $2 \mathrm{~N}$ ordinary differential equations with respect to the unknowns $\mathbf{U}(\mathbf{t})$ and $\mathbf{V}(\mathbf{t})$ :

$$
\frac{d U_{i}(t)}{d t}=V_{\hat{x}, i}, \quad \frac{d V_{i}(t)}{d t}=\left(E-\beta_{1} \hat{\Delta}_{h}\right)^{-1}\left(U_{\hat{x}, i}-\beta_{2} U_{\hat{x} \hat{x} \hat{x}, i}+(f(U))_{\hat{x}, i}\right) .
$$

Note that the new system (8) is also a generalized Hamiltonian system (or Poisson system) with a separable Hamiltonian $H_{h}(\mathbf{U}, \mathbf{V})$ and a matrix $J_{h}$ defined by

$$
H_{h}(\mathbf{U}, \mathbf{V})=\frac{1}{2} \sum_{i=1}^{N}\left(V_{i}^{2}+\beta_{1} V_{\hat{x}, i}^{2}+U_{i}^{2}+\beta_{2} U_{\hat{x}, i}^{2}+2 F\left(U_{i}\right)\right),
$$




$$
J_{h}=\left[\begin{array}{cc}
0 & \left(E-\beta_{1} \hat{\Delta_{h}}\right)^{-1} \partial_{\hat{x}} \\
\left(E-\beta_{1} \hat{\Delta_{h}}\right)^{-1} \partial_{\hat{x}} & 0
\end{array}\right] .
$$

Applying the staggered Störmer-Verlet method (equivalently the two-stage symplectic Lobatto IIIA-IIIB method, as in [1] for the Boussinesq equation) to (8), we obtain the following fully discrete system $F D S-S$ :

$$
\left\{\begin{array}{l}
\left(E-\beta_{1} \hat{\Delta}_{h}\right) \frac{V_{i}^{k+1 / 2}-V_{i}^{k-1 / 2}}{\tau}=U_{\hat{x}, i}^{k}-\beta_{2} U_{\hat{x} \hat{x} \hat{x}, i}^{k}+f\left(U_{i}^{k}\right)_{\hat{x}, i}, \\
\frac{U_{i}^{k+1}-U_{i}^{k}}{\tau}=V_{\hat{x}, i}^{k+1 / 2}
\end{array}\right.
$$

where $U_{i}^{k}$ and $V_{i}^{k+1 / 2}$ are approximations to $U_{i}$ and $V_{i}$ at time levels $t^{k}$ and $t^{k}+\tau / 2$, respectively.

We eliminate the function $V$ from (10); applying the operator $A=-\hat{\Delta}_{h}$ we get the 3-time level FDS that contains only the function $U_{i}^{k}$ :

$$
\left(E+\beta_{1} A\right) U_{\bar{t} t, i}^{k}+A U_{i}^{k}+\beta_{2} A^{2} U_{i}^{k}+A f\left(U_{i}^{k}\right)=0 .
$$

\subsection{Finite difference scheme FDS-M}

For the derivation of the new scheme, we keep the linear terms in (10) intact and only change the approximation to the nonlinear term $f(u)$. We consider the following FDS-M:

$$
\left\{\begin{array}{l}
\left(E-\beta_{1} \hat{\Delta}_{h}\right) \frac{V_{i}^{k+1 / 2}-V_{i}^{k-1 / 2}}{\tau}=U_{\hat{x}, i}^{k}-\beta_{2} U_{\hat{x} \hat{x} \hat{x}, i}^{k}+\left(\frac{F\left(U_{i+1}^{k}\right)-F\left(U_{i-1}^{k}\right)}{U_{i+1}^{k}-U_{i-1}^{k}}\right)_{\hat{x}, i}, \\
\frac{U_{i}^{k+1}-U_{i}^{k}}{\tau}=V_{\hat{x}, i}^{k+1 / 2} .
\end{array}\right.
$$

Eliminating the function $V$ from (12), one can obtain the equivalent FDS for $U_{i}^{k}$

$$
\left(E+\beta_{1} A\right) U_{\bar{t}, i}^{k}+A U_{i}^{k}+\beta_{2} A^{2} U_{i}^{k}+A\left(\frac{F\left(U_{i+1}^{k}\right)-F\left(U_{i-1}^{k}\right)}{U_{i+1}^{k}-U_{i-1}^{k}}\right)_{i}=0
$$

\subsection{Finite difference scheme FDS-E}

For consistency of presentation we also formulate the following well studied scheme (see e.g. $[17,19])$ :

$$
\left\{\begin{array}{l}
\left(E-\beta_{1} \hat{\Delta}_{h}\right) \frac{V_{i}^{k+1 / 2}-V_{i}^{k-1 / 2}}{\tau}=U_{\hat{x}, i}^{k}-\beta_{2} U_{\hat{x} \hat{x} \hat{x}, i}^{k}+\left(\frac{F\left(U_{i}^{k+1}\right)-F\left(U_{i}^{k-1}\right)}{U_{i}^{k+1}-U_{i}^{k-1}}\right) \hat{x}, i \\
\frac{U_{i}^{k+1}-U_{i}^{k}}{\tau}=V_{\hat{x}, i}^{k+1 / 2}
\end{array}\right.
$$

After eliminating the function $V$ from (14) we get the equivalent 3-time level FDS for function $U_{i}^{k}$

$$
\left(E+\beta_{1} A\right) U_{\bar{t}, i}^{k}+A U_{i}^{k}+\beta_{2} A^{2} U_{i}^{k}+A\left(\frac{F\left(U_{i}^{k+1}\right)-F\left(U_{i}^{k-1}\right)}{U_{i}^{k+1}-U_{i}^{k-1}}\right)_{i}=0 .
$$

\section{Analysis of finite difference schemes}

\subsection{Local approximation error, linear stability, implementation}

By Taylor series expansion at the point $\left(x_{i}, t^{k}\right)$ it is easy to conclude that both schemes FDS$S(11)$ and FDS-M (13) approximate the DDE with $O\left(h^{2}+\tau^{2}\right)$ local error. So the schemes are consistent with the DDE. 
The schemes FDS-S and FDS-M have the same linear part. Thus they have the same linear stability requirement.

Theorem 2 The linearized scheme corresponding to (11) (equivalently to (13)) is stable with respect to the initial data and the right-hand side if the steps $\tau$ and h satisfy the inequality

$$
\tau<h \sqrt{\frac{4 \beta_{1}}{(1+\epsilon)\left(\beta_{2}+h^{2}\right)}}
$$

for some positive number $\epsilon$, independent of $h, \tau$ and $U$.

The proof of this theorem follows by stability theory for FDS from [24] since the operator inequality $E+\beta_{1} A>\frac{1+\epsilon}{4}\left(A+\beta_{2} A^{2}\right)$ holds whenever the requirement (15) is satisfied.

Both schemes use nine mesh points of the stencil, thus they are non-compact. The schemes (10) and (12) are completely explicit, i.e. $V^{k+1 / 2}$ and $U^{k+1}$ can be directly evaluated from the corresponding systems if data $V^{k-1 / 2}$ and $U^{k}$ on the previous time level are known.

\subsection{Symplecticness}

The FDS (10) is symplectic by construction-the staggered Störmer-Verlet method (equivalently the two-stage symplectic Lobatto IIIA-IIIB method) is applied to the separable Hamiltonian system (8); see e.g. [14]. Thus the following result holds.

Theorem 3 The scheme FDS-S (10) is symplectic, i.e. it conserves the discrete symplectic structure $\omega^{k}=\mathbf{d} \mathbf{Z}^{\mathbf{k}} J_{h} \mathbf{d Z}^{\mathbf{k}-1}, \mathbf{Z}^{\mathbf{k}}=\left(U^{k}, V^{k-\frac{1}{2}}\right)$ at each time level: $\omega^{K}=\omega^{K-1}=\cdots=\omega^{1}$.

\subsection{Conservation of mass}

Let us define the discrete mass $I_{h}^{k}(U)$,

$$
I_{h}^{k}(U):=\sum_{i=1}^{N} h U_{i}^{k} .
$$

Note that the discrete mass (16) approximates the exact value of the mass (5) with $O\left(h^{2}\right)$ local error.

Theorem 4 All three schemes FDS-S (10), FDS-M (12) and FDS-E (14) conserve the discrete mass in time, i.e. the identity $I_{h}^{k}(U)=I_{h}^{0}(U)$ is true for all $k=1,2, \ldots K$.

Proof Under the periodic conditions imposed on the discrete functions $U, V$ and from the second equation of the corresponding systems (10), (12), and (14), the following identity holds:

$$
\frac{1}{\tau}\left(I_{h}^{k+1}(U)-I_{h}^{k}(U)\right)=\sum_{i=1}^{N} h \frac{U_{i}^{k+1}-U_{i}^{k}}{\tau}=\sum_{i=1}^{N} h V_{\hat{x}, i}^{k+1 / 2}=0 .
$$

Hence $I_{h}^{k+1}(U)=I_{h}^{k}(U)=\cdots=I_{h}^{0}(U)$ and Theorem 4 is proved. 


\subsection{Conservation of the discrete momentum}

Now we define the discrete momentum $M_{h}^{k}$ as

$$
M_{h}^{k}\left(U^{k}, V^{k-1 / 2}\right):=\sum_{i=1}^{N} h\left(U_{i}^{k} V_{i}^{k-1 / 2}+\beta_{1} U_{\hat{x}, i}^{k} V_{\hat{x}, i}^{k-1 / 2}\right)
$$

Note that $M_{h}^{k}\left(U^{k}, V^{k-1 / 2}\right)$ approximates the exact momentum (6) with $O\left(h^{2}+\tau^{2}\right)$ local error.

Theorem 5 (Momentum conservation law) The solution of the FDS-M (12) conserves the discrete momentum in time, i.e.

$$
M_{h}^{k}\left(U^{k}, V^{k-1 / 2}\right)=M_{h}^{1}\left(U^{1}, V^{1 / 2}\right), \quad k=2,3, \ldots, K .
$$

Proof We evaluate the difference $R$ between momentum at time levels $k+1$ and $k$ and obtain the equality

$$
\begin{aligned}
R & =\frac{1}{\tau}\left(M_{h}^{k+1}\left(U^{k+1}, V^{k+1 / 2}\right)-M_{h}^{k}\left(U^{k}, V^{k-1 / 2}\right)\right) \\
& =\frac{1}{\tau} \sum_{i=1}^{N} h\left(U_{i}^{k+1}\left(E-\beta_{1} \hat{\Delta}_{h}\right) V_{i}^{k+1 / 2}-U_{i}^{k}\left(E-\beta_{1} \hat{\Delta}_{h}\right) V_{i}^{k-1 / 2}\right) .
\end{aligned}
$$

After adding and subtracting the term $U_{i}^{k}\left(I-\beta_{1} \hat{\Delta}_{h}\right) V_{i}^{k+1 / 2}$ to the right-hand side of $R$ we get

$$
\begin{aligned}
R= & \sum_{i=1}^{N} h\left(U_{t, i}^{k}\left(I-\beta_{1} \hat{\Delta}_{h}\right) V_{i}^{k+1 / 2}+U_{i}^{k}\left(I-\beta_{1} \hat{\Delta}_{h}\right) V_{t, i}^{k-1 / 2}\right) \\
= & \sum_{i=1}^{N} h V_{\hat{x}, i}^{k+1 / 2}\left(I-\beta_{1} \hat{\Delta}_{h}\right) V_{i}^{k+1 / 2} \\
& +\sum_{i=1}^{N} h U_{i}^{k}\left(U_{\hat{x}, i}^{k}-\beta_{2} U_{\hat{x} \hat{x} \hat{x}, i}^{k}+\left(\frac{F\left(U_{i+1}^{k}\right)-F\left(U_{i-1}^{k}\right)}{U_{i+1}^{k}-U_{i-1}^{k}}\right)_{\hat{x}, i}\right) \\
= & \sum_{i=1}^{N} h V_{\hat{x}, i}^{k+1 / 2} V_{i}^{k+1 / 2}+\beta_{1} \sum_{i=1}^{N} h V_{\hat{x} \hat{x}, i}^{k+1 / 2} V_{\hat{x}, i}^{k+1 / 2}+\sum_{i=1}^{N} h U_{i}^{k}\left(U_{\hat{x}, i}^{k}-\beta_{2} U_{\hat{x} \hat{x}, i}^{k}\right) \\
& +\sum_{i=1}^{N} h U_{i}^{k}\left(\frac{F\left(U_{i+1}^{k}\right)-F\left(U_{i-1}^{k}\right)}{U_{i+1}^{k}-U_{i-1}^{k}}\right)_{\hat{x}, i} \\
= & 0 .
\end{aligned}
$$

All terms in the previous equality are equal to zero because of the periodicity of the discrete functions. Additionally, the last term in the previous equality is zero because of the 
following transformations:

$$
\begin{aligned}
& \sum_{i=1}^{N} h U_{i}^{k}\left(\frac{F\left(U_{i+1}^{k}\right)-F\left(U_{i-1}^{k}\right)}{U_{i+1}^{k}-U_{i-1}^{k}}\right)_{\hat{x}, i} \\
& =-\sum_{i=1}^{N} h\left(U_{i}^{k}\right)_{\hat{x}, i}\left(\frac{F\left(U_{i+1}^{k}\right)-F\left(U_{i-1}^{k}\right)}{U_{i+1}^{k}-U_{i-1}^{k}}\right) \\
& =-\frac{1}{2} \sum_{i=1}^{N}\left(U_{i+1}^{k}-U_{i-1}^{k}\right) \frac{F\left(U_{i+1}^{k}\right)-F\left(U_{i-1}^{k}\right)}{U_{i+1}^{k}-U_{i-1}^{k}}=-\frac{1}{2} \sum_{i=1}^{N}\left(F\left(U_{i+1}^{k}\right)-F\left(U_{i-1}^{k}\right)\right)=0 .
\end{aligned}
$$

Thus $M_{h}^{k+1}\left(U^{k+1}, V^{k+1 / 2}\right)=M_{h}^{k}\left(U^{k}, V^{k-1 / 2}\right)$ for every $k=1,2, \ldots$, which completes the proof of Theorem 5 .

The preservation of the discrete momentum $M_{h}^{k}$ by the scheme FDS-S (10) is examined in the next theorem.

Theorem 6 (Discrete identity for momentum) The solution of FDS-S (10) satisfies the following discrete identities between every two consecutive time levels $k+1$ and $k$ :

$$
M_{h}^{k+1}\left(U^{k+1}, V^{k+1 / 2}\right)-M_{h}^{k}\left(U^{k}, V^{k-1 / 2}\right)=-\tau \sum_{i=1}^{N} h U_{\hat{x}, i}^{k} f\left(U_{i}^{k}\right), \quad k=1,2, \ldots, K-1 .
$$

Moreover,

$$
\left|M_{h}^{K}\left(U^{K}, V^{K-1 / 2}\right)-M_{h}^{1}\left(U^{1}, V^{1 / 2}\right)\right| \leq C_{1} h^{2} T .
$$

Proof We study the difference $M_{h}^{k+1}\left(U^{k+1}, V^{k+1 / 2}\right)-M_{h}^{k}\left(U^{k}, V^{k-1 / 2}\right)$ on the solution of (10). The treatment of all the terms but the nonlinear one is the same as in the proof of Theorem 5. We have

$$
M_{h}^{k+1}\left(U^{k+1}, V^{k+1 / 2}\right)-M_{h}^{k}\left(U^{k}, V^{k-1 / 2}\right)=-\tau \sum_{i=1}^{N} h U_{\hat{x}, i}^{k} f\left(U_{i}^{k}\right),
$$

which proves (18).

We add the term $\tau \sum_{i=1}^{N} h U_{\hat{x}, i}^{k}\left(\frac{F\left(U_{i+1}^{k}\right)-F\left(U_{i-1}^{k}\right)}{U_{i+1}^{k}-U_{i-1}^{k}}\right)=0$ to the right-hand side of (20) and estimate the expression $R^{k}$

$$
\begin{aligned}
R^{k} & :=M_{h}^{k+1}\left(U^{k+1}, V^{k+1 / 2}\right)-M_{h}^{k}\left(U^{k}, V^{k-1 / 2}\right) \\
& =\tau \sum_{i=1}^{N} h U_{\hat{x}, i}^{k}\left(\frac{F\left(U_{i+1}^{k}\right)-F\left(U_{i-1}^{k}\right)}{U_{i+1}^{k}-U_{i-1}^{k}}-f\left(U_{i}^{k}\right)\right) .
\end{aligned}
$$

Using the definition of the nonlinearity functions $f$ and $F$, we get

$$
\begin{aligned}
S_{i}^{k} & :=\frac{F\left(U_{i+1}^{k}\right)-F\left(U_{i-1}^{k}\right)}{U_{i+1}^{k}-U_{i-1}^{k}}-f\left(U_{i}^{k}\right) \\
& =\frac{\alpha}{p+1}\left(\left(U_{i+1}^{k}\right)^{p}+\left(U_{i+1}^{k}\right)^{p-1} U_{i-1}^{k}+\cdots+\left(U_{i-1}^{k}\right)^{p}-(p+1)\left(U_{i}^{k}\right)^{p}\right) .
\end{aligned}
$$


Applying a Taylor series expansion about the point $x_{i}$, we obtain the estimate

$$
\left|S_{i}^{k}\right| \leq h^{2} \frac{\alpha}{p+1} C_{2, i}
$$

where the constant $C_{2, i}$ depends on the values of the function $u$ and its first and second derivatives, i.e. $C_{2, i}=C_{2, i}\left(\max _{\xi \in\left(x_{i-1}, x_{i+1}\right)}\left\{\left|u\left(\xi, t^{k}\right)\right|,\left|\frac{\partial u\left(\xi, t^{k}\right)}{\partial x}\right|,\left|\frac{\partial^{2} u\left(\xi, t^{k}\right)}{\partial x^{2}}\right|\right\}\right)$. We substitute the estimate (23) into (21) to get

$$
\left|R^{k}\right| \leq \tau h^{2} \frac{\alpha}{p+1} \sum_{i=1}^{N} h\left|U_{\hat{x}, i}^{k}\right| C_{2, i} \leq \tau h^{2} \frac{2 \alpha}{p+1} C_{3} .
$$

We add equalities (21) for $k=1,2, \ldots, K-1$, apply (24), and obtain

$$
\begin{aligned}
M_{h}^{K}\left(U^{K}, V^{K-1 / 2}\right)-M_{h}^{1}\left(U^{1}, V^{1 / 2}\right) & =\sum_{k=1}^{K-1} R^{k} \\
\left|M_{h}^{K}\left(U^{K}, V^{K-1 / 2}\right)-M_{h}^{1}\left(U^{1}, V^{1 / 2}\right)\right| & \leq \sum_{k=1}^{K-1}\left|R^{k}\right| \leq \sum_{k=1}^{K-1} \tau h^{2} \frac{2 \alpha}{p+1} C_{4} \\
& \leq K \tau h^{2} \frac{2 \alpha}{p+1} C_{4} \leq h^{2} T C_{1},
\end{aligned}
$$

which completes the proof of the estimate (19) and of Theorem 6.

In conclusion to this subsection, we see that the FDS-M (12) conserves the discrete momentum exactly in time, which fully corresponds to the conservation of the exact momentum (6).

The scheme FDS-S (10) does not strictly conserve the discrete momentum $M_{h}^{k}$ but it preserves the discrete momentum approximately with $O\left(h^{2}\right)$ global error.

Remark 1 Following the lines of the proof of Theorem 6, we can prove that the solution to the FDS-E conserves the discrete momentum approximately with $O\left(h^{2}\right)$ global error.

\subsection{Conservation of the discrete Hamiltonian (energy)}

Following the results from $[17,19]$ we define the linear part of the discrete Hamiltonian $H_{h, L}^{k}$ as

$$
\begin{aligned}
H_{h, L}^{k}\left(U^{k}, V^{k+1 / 2}\right):= & \frac{1}{2}\left\|V^{k+1 / 2}\right\|^{2}+\left(\frac{\beta_{1}}{2}-\frac{\tau^{2}}{8}\right)\left\|V_{\hat{x}}^{k+1 / 2}\right\|^{2}-\frac{\beta_{2} \tau^{2}}{8}\left\|V_{\hat{x} \hat{x}}^{k+1 / 2}\right\|^{2} \\
& +\frac{1}{8}\left\|U^{k+1}+U^{k}\right\|^{2}+\frac{\beta_{2}}{8}\left\|U_{\hat{x}}^{k+1}+U_{\hat{x}}^{k}\right\|^{2} .
\end{aligned}
$$

By incorporating the nonlinearity we consider the full discrete Hamiltonian

$$
H_{h}^{k}\left(U^{k}, V^{k+1 / 2}\right):=H_{h, L}^{k}\left(U^{k}, V^{k+1 / 2}\right)+\frac{1}{2}\left\langle F\left(U^{k+1}\right)+F\left(U^{k}\right), 1\right\rangle .
$$

Note that the discrete Hamiltonian $H_{h}^{k}$ approximates the exact Hamiltonian $H(U, V)$ given in (3) with $O\left(h^{2}+\tau^{2}\right)$ local error.

For completeness of the presentation we state the following theorem (see $[17,19])$. 
Theorem 7 (Conservation of the discrete Hamiltonian) The solution to the FDS-E (14) conserves the full discrete energy $H_{h}^{k}$ in time, i.e.

$$
H_{h}^{k}\left(U^{k}, V^{k+1 / 2}\right)=H_{h}^{0}\left(U^{0}, V^{1 / 2}\right), \quad k=1,2, \ldots, K .
$$

In the next theorem we study the preservation of the discrete Hamiltonian (26) over the solutions of FDS-S and FDS-M.

\section{Theorem 8}

(i) The solution to the FDS-S (10) satisfies the following identities at each time level $k=1,2, \ldots, K$ :

$$
H_{h, L}^{k}\left(U^{k}, V^{k+1 / 2}\right)=H_{h, L}^{k-1}\left(U^{k-1}, V^{k-1 / 2}\right)+0.5\left\langle f\left(U^{k}\right), U^{k+1}-U^{k-1}\right\rangle .
$$

Moreover, it conserves approximately with $O\left(\tau^{2}\right)$ global error the discrete Hamiltonian $H_{h}^{0}\left(U^{0}, V^{1 / 2}\right)$, i.e.

$$
\left|H_{h}^{K}\left(U^{K}, V^{K+1 / 2}\right)-H_{h}^{0}\left(U^{0}, V^{1 / 2}\right)\right| \leq \tau^{2} T C_{5} .
$$

(ii) The solution to the FDS-M (12) satisfies the following identities at each time level $k=1,2, \ldots, K$ :

$$
\begin{aligned}
H_{h, L}^{k}\left(U^{k}, V^{k+1 / 2}\right)= & H_{h, L}^{k-1}\left(U^{k-1}, V^{k-1 / 2}\right) \\
& +\sum_{i=1}^{N} h \frac{F\left(U_{i+1}^{k}\right)-F\left(U_{i-1}^{k}\right)}{U_{i+1}^{k}-U_{i-1}^{k}}\left(U_{i}^{k+1}-U_{i}^{k-1}\right) .
\end{aligned}
$$

Moreover, it preserves approximately with $O\left(h^{2}+\tau^{2}\right)$ global error the discrete Hamiltonian $H_{h}^{0}\left(U^{0}, V^{1 / 2}\right)$, i.e.

$$
\left|H_{h}^{K}\left(U^{K}, V^{K+1 / 2}\right)-H_{h}^{0}\left(U^{0}, V^{1 / 2}\right)\right| \leq\left(h^{2}+\tau^{2}\right) T C_{6} .
$$

Proof Applying $A^{-1}$ to (11) we get

$$
A^{-1}\left(E+\beta_{1} A\right) U_{\bar{t} t}^{k}+\left(E+\beta_{2} A\right) U^{k}+f\left(U^{k}\right)=0
$$

We multiply the last equality by $\frac{u_{i}^{k+1}-U_{i}^{k-1}}{2}=\frac{\tau\left(U_{t, i}^{k}+U_{t, i}^{k}\right)}{2}$, substitute the following expressions:

$$
U_{\bar{t} t}=\frac{U_{t}-U_{\bar{t}}}{\tau}, \quad U^{k}=\frac{U^{k+1}+U^{k-1}}{2}-\frac{\tau}{2}\left(U_{t}^{k}-U_{\bar{t}}^{k}\right)
$$

and sum these equalities for $i=1$ to $i=N$. We obtain

$$
\begin{aligned}
& \frac{1}{2}\left\langle\left(A^{-1}+\beta_{1} E-\frac{\tau^{2}}{4} E-\frac{\beta_{2} \tau^{2} A}{4}\right) U_{t}^{k}, U_{t}^{k}\right\rangle+\frac{1}{8}\left\|U^{k+1}+U^{k}\right\|^{2} \\
& \quad+\frac{\beta_{2}}{8}\left\langle A\left(U^{k+1}+U^{k}\right), U^{k+1}+U^{k}\right\rangle
\end{aligned}
$$




$$
\begin{aligned}
& -\frac{1}{2}\left\langle\left(A^{-1}+\beta_{1} E-\frac{\tau^{2}}{4} E-\frac{\beta_{2} \tau^{2} A}{4}\right) U_{t}^{k-1}, U_{t}^{k-1}\right\rangle-\frac{1}{8}\left\|U^{k}+U^{k-1}\right\|^{2} \\
& -\frac{\beta_{2}}{8}\left\langle A\left(U^{k}+U^{k-1}\right), U^{k}+U^{k-1}\right\rangle+\frac{1}{2}\left\langle f\left(U^{k}\right), U^{k+1}-U^{k-1}\right\rangle=0 .
\end{aligned}
$$

Using definition (25) and the relations $\left(A^{-1} U_{t}, U_{t}\right)=\left(V^{k+1 / 2}, V^{k+1 / 2}\right), U_{t}^{k}=V_{\hat{x}}^{k+1 / 2}$, we get the identity (28).

In view of the equalities (27) and (28), one has to estimate from above the expression $R^{k}$

$$
\begin{aligned}
R^{k} & :=H_{h}^{k}\left(U^{k}, V^{k+1 / 2}\right)-H_{h}^{k-1}\left(U^{k-1}, V^{k-1 / 2}\right) \\
& =\frac{1}{2}\left\langle f\left(U^{k}\right), U^{k+1}-U^{k-1}\right\rangle-\frac{1}{2}\left\langle F\left(U^{k+1}\right)-F\left(U^{k-1}\right), 1\right\rangle .
\end{aligned}
$$

We reorganize $R^{k}$ using the definitions of functions $f$ and $F$ to get

$$
\begin{aligned}
R^{k}= & \frac{1}{2} \frac{\alpha}{p+1}\left\langle U^{k+1}-U^{k-1},\right. \\
& \left.(p+1)\left(U^{k}\right)^{p}-\left(U^{k+1}\right)^{p}-\left(U^{k+1}\right)^{p-1} U^{k-1}-\cdots-\left(U^{k-1}\right)^{p}\right\rangle .
\end{aligned}
$$

The right-hand side term in the previous equality is very similar to the term $S_{i}^{k}$ in (22), which was studied thoroughly in the proof of Theorem 6 . Therefore we get the similar to (23) estimate

$$
\left|R^{k}\right| \leq \frac{1}{2} \frac{\alpha}{p+1} \tau^{2}|| U^{k+1}-U^{k-1}|, 1\rangle \leq \tau^{3}\left(\left\|U_{t}^{k}\right\|_{L_{1, h}}+\left\|U_{t}^{k}\right\|_{L_{1, h}}\right) C_{7}<C_{8} \tau^{3},
$$

i.e. the error $H_{h}^{k}\left(U^{k}, V^{k+1 / 2}\right)-H_{h}^{k-1}\left(U^{k-1}, V^{k-1 / 2}\right)$ between two consecutive values of the Hamiltonian is estimated from above by $O\left(\tau^{3}\right)$.

We proceed as in the proof of Theorem 6, i.e. we sum the equalities (29) for $k=1$ to $K$ to obtain the difference between the Hamiltonian at the last time $K$ and the Hamiltonian at the initial moment $H_{h}^{K}\left(U^{K}, V^{K+1 / 2}\right)-H_{h}^{0}\left(U^{0}, V^{1 / 2}\right)$. Summing the errors between two consecutive levels obtained in (30), we get the overall error of order $O\left(K \tau^{3}\right)=O\left(T \tau^{2}\right)$ and complete the proof of statement (i).

The proof of statement (ii) goes the same way as the proof of Theorem 8(i) and therefore we omit it.

We conclude that schemes FDS-S and FDS-M both conserve the discrete Hamiltonian approximately with $O\left(h^{2}+\tau^{2}\right)$ global error.

\section{Numerical results and discussion}

In this section we present numerical results about the properties of the schemes FDS-S and $F D S-M$. Nested grids are used to estimate the accuracy and the convergence of the numerical solution to the exact one, as well as the behavior of the discrete momentum and the discrete energy. We analyze numerically the preservation of the discrete energy, computed by Eq. (26), and the discrete momentum, evaluated by (17).

In all computations the discrete mass (16) is preserved with $O\left(10^{-14}\right)$ error. Therefore the results about the mass conservation are not included in the tables.

All numerical results are obtained in the case of quadratic nonlinearity $\left(f(u)=\alpha u^{2}\right)$. The proposed schemes are tested on two problems typical for the DDE: 
Problem 1 (Propagation of a solitary wave) Let the parameters of the problem (4) be $\alpha=3$, $\beta_{1}=1.5, \beta_{2}=0.5, c=2$, and the initial conditions be

$$
u(x, 0)=\varphi(x, 0 ; c), \quad v\left(x, \frac{\tau}{2}\right)=-c \varphi\left(x, \frac{\tau}{2} ; c\right) .
$$

Here $\varphi(x, t ; c)$ is the exact solitary wave solution given by $(7)$. The propagation of the numerical solution in time (obtained by FDS-S) is plotted on Fig. 1.

Table 1 and Table 2 show the numerical results for the scheme FDS-S and for the scheme FDS- $M$, respectively. The numerical parameters of the FDS are $x \in[-80,80], T=20$, $t \in[0, T]$. The maximal differences $\psi_{h}$ between the exact solution (7) and the numerical solutions are given in the column Error $\psi_{h}$. The order of convergence is calculated by $\kappa=\log _{2}\left(\frac{\psi_{h}}{\psi_{h / 2}}\right)$.

In the columns Energy the discrete energy at the final time $H_{h}^{K}$ and the absolute value of the difference between the initial energy $H_{h}^{0}$ and the final energy $H_{h}^{K},\left|H_{h}^{0}-H_{h}^{K}\right|$ are given.

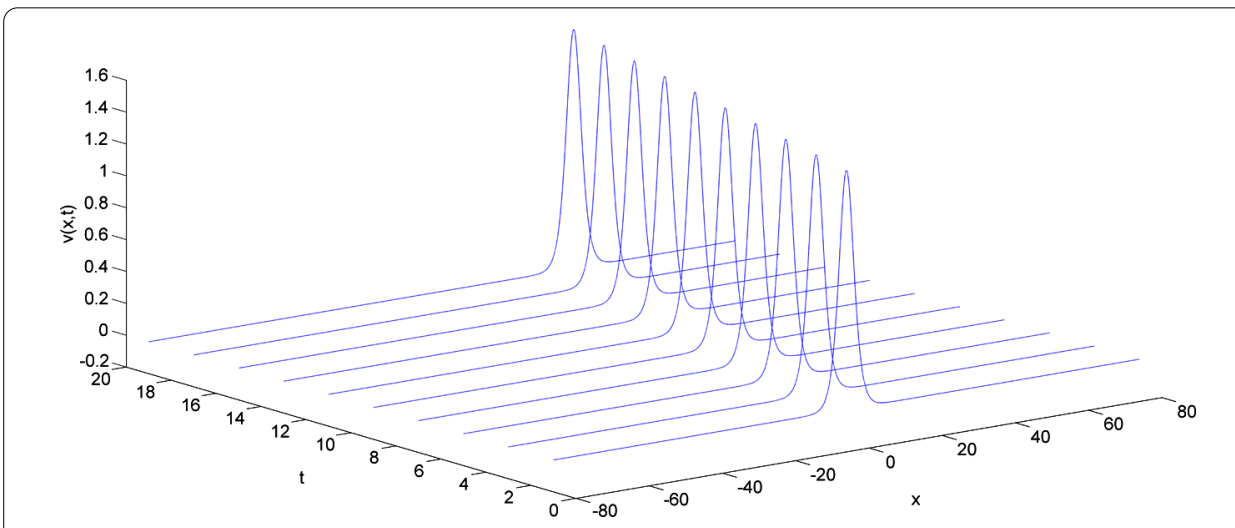

Figure 1 The propagation of one solitary wave

Table 1 Error, energy and momentum for Problem 1 using FDS-S

\begin{tabular}{|c|c|c|c|c|c|c|}
\hline \multirow[t]{2}{*}{$\overline{h=\tau}$} & \multicolumn{2}{|l|}{ Error } & \multicolumn{2}{|l|}{ Energy } & \multicolumn{2}{|l|}{ Momentum } \\
\hline & $\overline{\psi_{h}}$ & $\kappa$ & $\overline{H_{h}^{K}}$ & $\left|H_{h}^{0}-H_{h}^{K}\right|$ & $\overline{M_{h}^{K}}$ & $\left|M_{h}^{0}-M_{h}^{K}\right|$ \\
\hline$\overline{0.1}$ & $3.5 * 10^{-3}$ & & 32.92502487 & $4.9 * 10^{-5}$ & -18.88936152 & $1.9 * 10^{-5}$ \\
\hline 0.05 & $8.6 * 10^{-4}$ & 2.00 & 32.93570500 & $3.1 * 10^{-6}$ & -18.90247949 & $1.2 * 10^{-6}$ \\
\hline 0.025 & $2.1 * 10^{-4}$ & 2.00 & 32.93838765 & $1.9 * 10^{-7}$ & -18.90575959 & $7.5 * 10^{-8}$ \\
\hline 0.0125 & $5.4 * 10^{-5}$ & 2.00 & 32.93905910 & $1.1 * 10^{-8}$ & -18.90657966 & $4.3 * 10^{-9}$ \\
\hline 0.00625 & $1.3 * 10^{-5}$ & 2.00 & 32.93922701 & $8.1 * 10^{-11}$ & -18.90678468 & $1.2 * 10^{-10}$ \\
\hline
\end{tabular}

Table 2 Error, energy and momentum for Problem 1 using FDS-M

\begin{tabular}{|c|c|c|c|c|c|c|}
\hline \multirow[t]{2}{*}{$h=\tau$} & \multicolumn{2}{|l|}{ Error } & \multicolumn{2}{|l|}{ Energy } & \multicolumn{2}{|l|}{ Momentum } \\
\hline & $\overline{\psi_{h}}$ & $\kappa$ & $\overline{H_{h}^{K}}$ & $\left|H_{h}^{0}-H_{h}^{K}\right|$ & $\overline{M_{h}^{K}}$ & $\left|M_{h}^{0}-M_{h}^{K}\right|$ \\
\hline 0.1 & $8.0 * 10^{-3}$ & & 32.92488059 & $5.4 * 10^{-5}$ & -18.88938087 & $4.3 * 10^{-10}$ \\
\hline 0.05 & $2.0 * 10^{-3}$ & 2.00 & 32.93569601 & $3.4 * 10^{-6}$ & -18.90248070 & $4.2 * 10^{-10}$ \\
\hline 0.025 & $5.0 * 10^{-4}$ & 2.00 & 32.93838709 & $2.2 * 10^{-7}$ & -18.90575967 & $4.3 * 10^{-10}$ \\
\hline 0.0125 & $1.3 * 10^{-4}$ & 2.00 & 32.93905907 & $1.4 * 10^{-8}$ & -18.90657966 & $4.4 * 10^{-10}$ \\
\hline 0.00625 & $3.1 * 10^{-5}$ & 2.00 & 32.93922701 & $1.8 * 10^{-9}$ & -18.90678468 & $4.9 * 10^{-10}$ \\
\hline
\end{tabular}


Similarly we give the discrete momentum at the final time $M_{h}^{K}$ and the error between the values of the momentum at the initial and the final time, $\left|M_{h}^{0}-M_{h}^{K}\right|$, in the last two columns.

The numerical results show that the computed solution is very close to the exact one with a maximal error $\psi_{h}$ of order $10^{-5}$ for the smallest steps $h=\tau=0.00625$. The presented experiments show second order of convergence for both schemes.

Problem 2 (Interaction of two solitary waves) In this example we simulate interaction of two solitary waves running in opposite directions with different velocities, $c_{1}=1.1$ and $c_{2}=-1.3$. The initial data of problem (4) are

$$
\begin{aligned}
& u(x, 0)=\varphi\left(x+30,0 ; c_{1}\right)+\varphi\left(x-40,0 ; c_{2}\right), \\
& v\left(x, \frac{\tau}{2}\right)=-c_{1} \varphi\left(x+30, \frac{\tau}{2} ; c_{1}\right)-c_{2} \varphi\left(x-40, \frac{\tau}{2} ; c_{2}\right),
\end{aligned}
$$

and $\alpha=3, \beta_{1}=1.5, \beta_{2}=0.5$. The snapshots of the numerical solution (obtained by FDS-M) at different evolution times are plotted on Fig. 2.

Table 3 and Table 4 show the numerical results for the scheme FDS-S and for the scheme $F D S-M$, for $x \in[-160,160]$ and $T=80$. There is no exact solution to Problem 2, therefore the error $\psi_{h}$ and the order of convergence $\kappa$ are evaluated by the following expres-

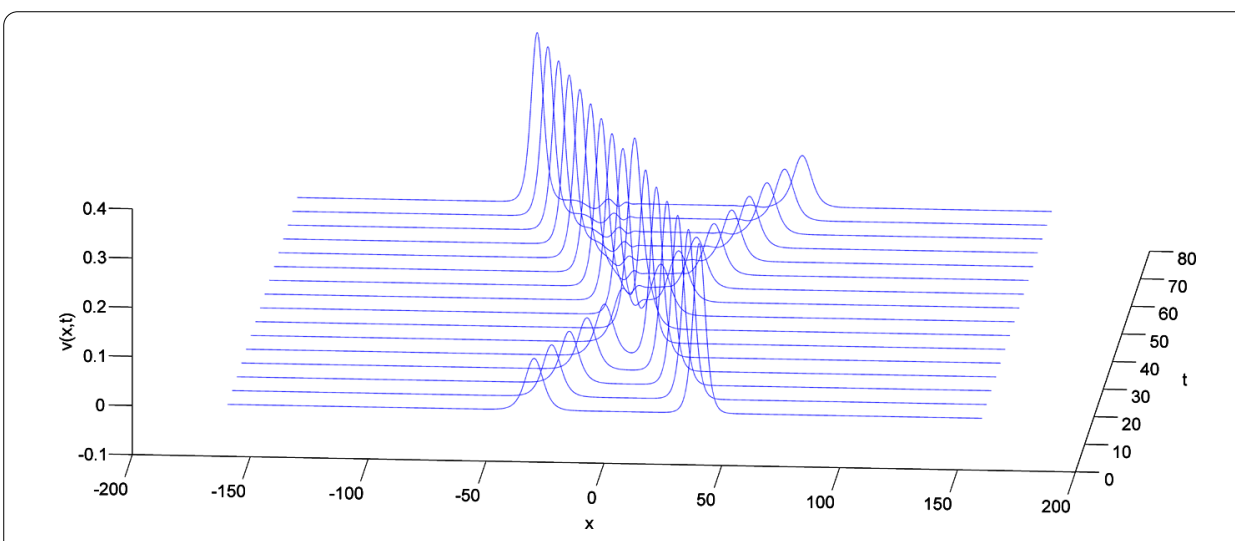

Figure 2 The interaction of two solitary waves

\begin{tabular}{|c|c|c|c|c|c|c|}
\hline \multirow[t]{2}{*}{$\bar{h}=\tau$} & \multicolumn{2}{|l|}{ Error } & \multicolumn{2}{|l|}{ Energy } & \multicolumn{2}{|l|}{ Momentum } \\
\hline & $\overline{\psi_{h}}$ & $\kappa$ & $\overline{H_{h}^{K}}$ & $\left|H_{h}^{0}-H_{h}^{K}\right|$ & $\overline{M_{h}^{K}}$ & $\left|M_{h}^{0}-M_{h}^{K}\right|$ \\
\hline 0.4 & & & 1.02626203 & $3.6 * 10^{-6}$ & 0.69289524 & $2.9 * 10^{-5}$ \\
\hline 0.2 & & & 1.02895892 & $4.0 * 10^{-7}$ & 0.69512790 & $1.4 * 10^{-6}$ \\
\hline 0.1 & $1.1 * 10^{-2}$ & 2.00 & 1.02964231 & $7.5 * 10^{-8}$ & 0.69569642 & $1.4 * 10^{-8}$ \\
\hline 0.05 & $2.6 * 10^{-3}$ & 2.00 & 1.02981375 & $1.7 * 10^{-8}$ & 0.69583920 & $2.7 * 10^{-8}$ \\
\hline 0.025 & $6.6 * 10^{-4}$ & 2.00 & 1.02985665 & $4.2 * 10^{-9}$ & 0.69587494 & $8.1 * 10^{-9}$ \\
\hline 0.0125 & $1.6 * 10^{-4}$ & 2.00 & 1.02986738 & $1.0 * 10^{-9}$ & 0.69588387 & $2.1 * 10^{-9}$ \\
\hline 0.00625 & $4.1 * 10^{-5}$ & 2.00 & 1.02987006 & $3.1 * 10^{-10}$ & 0.69588611 & $4.9 * 10^{-10}$ \\
\hline
\end{tabular}

Table 3 Error, energy and momentum for Problem 2 using FDS-S 
Table 4 Error, energy and momentum for Problem 2 using FDS-M

\begin{tabular}{|c|c|c|c|c|c|c|}
\hline \multirow[t]{2}{*}{$h=\tau$} & \multicolumn{2}{|l|}{ Error } & \multicolumn{2}{|l|}{ Energy } & \multicolumn{2}{|l|}{ Momentum } \\
\hline & $\psi_{h}$ & $\kappa$ & $\overline{H_{h}^{K}}$ & $\left|H_{h}^{0}-H_{h}^{K}\right|$ & $M_{h}^{K}$ & $\left|M_{h}^{0}-M_{h}^{K}\right|$ \\
\hline$\overline{0.4}$ & & & 1.02593880 & $1.7 * 10^{-4}$ & 0.69286589 & $2.8 * 10^{-13}$ \\
\hline 0.2 & & & 1.02893447 & $1.4 * 10^{-5}$ & 0.69512648 & $2.9 * 10^{-13}$ \\
\hline 0.1 & $4.0 * 10^{-2}$ & 1.92 & 1.02964003 & $1.6 * 10^{-6}$ & 0.69569643 & $2.9 * 10^{-13}$ \\
\hline 0.05 & $1.1 * 10^{-2}$ & 1.98 & 1.02981343 & $2.7 * 10^{-7}$ & 0.69583923 & $3.8 * 10^{-13}$ \\
\hline 0.025 & $2.7 * 10^{-3}$ & 1.99 & 1.02985658 & $6.0 * 10^{-8}$ & 0.69587494 & $2.1 * 10^{-12}$ \\
\hline 0.0125 & $6.7 * 10^{-4}$ & 2.00 & 1.02986736 & $1.4 * 10^{-8}$ & 0.69588388 & $7.5 * 10^{-12}$ \\
\hline 0.00625 & $1.7 * 10^{-4}$ & 2.00 & 1.02987005 & $3.5 * 10^{-9}$ & 0.69588611 & $3.7 * 10^{-11}$ \\
\hline
\end{tabular}

sions:

$$
\psi_{h / 4}=\frac{\left(\left\|U_{[h]}-U_{[h / 2]}\right\|\right)^{2}}{\left\|U_{[h]}-U_{[h / 2]}\right\|-\left\|U_{[h / 2]}-U_{[h / 4]}\right\|}, \quad \kappa=\log _{2}\left(\frac{\left\|U_{[h]}-U_{[h / 2]}\right\|}{\left\|U_{[h / 2]}-U_{[h / 4]}\right\|}\right) .
$$

The remaining quantities in Table 3 and Table 4 are the same as in Table 1 and Table 2 .

The results about the error and the order of convergence are similar to those in Problem 1 . The maximal error $\psi_{h}$ is of order $10^{-5}$ for FDS-S and $10^{-4}$ for FDS-M with steps $h=\tau=0.00625$. The energy is preserved approximately up to $10^{-10}$ for FDS-S and up to $10^{-9}$ for FDS-M.

The calculations given in Table 1 through Table 4 demonstrate the second rate of convergence $O\left(h^{2}+\tau^{2}\right)$ of the discrete solution obtained by FDS-S or by FDS-M to the exact solution.

The scheme FDS-M conserves the discrete momentum with $O\left(10^{-10}\right)$ accuracy for solution of Problem 1 and up to $O\left(10^{-13}\right)$ for the solution of Problem 2.

The discrete energy is preserved with error $O\left(10^{-8}\right)$ for Problem 1 at final time $T=20$ and with error $O\left(10^{-10}\right)$ for Problem 2 at final time $T=80$.

Moreover, the analysis of the errors $\left|M_{h}^{0}-M_{h}^{K}\right|$ and $\left|H_{h}^{0}-H_{h}^{K}\right|$ on nested meshes shows that these errors decrease four times between meshes with step $h=\tau$ and $\frac{h}{2}=\frac{\tau}{2}$. Thus the results in Theorem 6 and Theorem 8 for approximate conservation of the discrete momentum and the discrete energy with $O\left(h^{2}+\tau^{2}\right)$ global error are confirmed numerically.

Remark 2 As a first step in the construction of our symplectic FDS-S we approximate the continuous Hamiltonian system (4) by the semi-discrete in space Hamiltonian system (8). The discrete mass

$$
\tilde{I}_{h}^{t}(\mathbf{U})=\sum_{i=1}^{N} h U_{i}(t)
$$

and the semi-discrete Hamiltonian (9) are invariants of (8). It is well-known, that linear first integrals and some quadratic first integrals of a special form are exactly preserved by the Störmer-Verlet method (see e.g. [13, Theorems 3.3 and 3.5]), while, in general, quadratic first integrals are not exactly preserved by the same method (see [13, Example 3.4]). 
In our case the discrete mass is a linear first integral and, hence, it is exactly preserved (see Theorem 4). The semi-discrete Hamiltonian (9) is not a quadratic invariant of the mentioned above special form; moreover, it is nonlinear w.r.t. $U_{i}$ due to the nonlinearity $F\left(U_{i}\right)$. Thus, one should not expect the exact preservation of the Hamiltonian (9). However, we prove in Theorem 8 that it is approximately preserved with a $O\left(\tau^{2}\right)$ global error.

A natural approximation to the continuous momentum (6) on the semi-discrete level is

$$
\tilde{M}_{h}(\mathbf{U}, \mathbf{V})(t)=\sum_{i=1}^{N} h\left\{U_{i}(t) V_{i}(t)+\beta_{1} U_{\hat{x}, i}(t) V_{\hat{x}, i}(t)\right\}
$$

Direct calculations show that $\tilde{M}_{h}$ is not an invariant of the system (8) due to the necessity to approximate the nonlinear term $\partial_{x} f(u)$ in (4). Hence, we do not expect that the semidiscrete momentum (31) will be exactly preserved after applying a symplectic integration method to (8). However, we prove in Theorem 6 that the solution to the symplectic scheme FDS-S approximately preserves the discrete momentum (17) with $O\left(h^{2}\right)$ global error.

\section{Conclusion}

Two new finite difference schemes for double dispersion equations are introduced and studied. The schemes give second order of approximation to the exact solution at the final time $T$. The linear stability requirement is not restrictive-it is $\tau=O(h)$ (see (15)). The algorithm for evaluation of the numerical solution is explicit.

The scheme FDS-S preserves exactly the symplectic structure of the discrete solution and the discrete mass and conserves approximately with $O\left(h^{2}+\tau^{2}\right)$ global error the discrete momentum and the discrete energy. The scheme FDS-M preserves exactly the discrete momentum and the discrete mass and approximately with $O\left(h^{2}+\tau^{2}\right)$ global error the discrete energy.

Note that the discrete mass is preserved exactly by both FDS. Moreover, the schemes show good long time behavior.

The two new schemes are also compared to the early studied scheme FDS-E, which preserves exactly the discrete energy and the discrete mass.

This way we are given a variety of FDS, such that each one of them preserves two of the discrete invariants exactly and approximately with global error $O\left(h^{2}+\tau^{2}\right)$ the remaining invariants. 


\section{Publisher's Note}

Springer Nature remains neutral with regard to jurisdictional claims in published maps and institutional affiliations.

\section{Received: 20 August 2018 Accepted: 21 May 2019 Published online: 31 May 2019}

\section{References}

1. Aydin, A., Karasözen, B.: Symplectic and multisymplectic Lobato methods for the 'good' Boussinesq equation. J. Math. Phys. 49, 083509 (2008)

2. Bratsos, A.: A second order numerical scheme for the solution of the one-dimensional Boussinesq equation. Numer. Algorithms 46, 45-58 (2007)

3. Brugnano, L., Gurioli, G., Zhang, C.: Spectrally accurate energy-preserving methods for the numerical solution of the "good" Boussinesq equation. Numer. Methods Partial Differ. Equ. 35(4), 1343-1362 (2019)

4. Cai, J., Wang, Y.: Local structure-preserving algorithms for the "good" Boussinesq equation. J. Comput. Phys. 239, $72-89$ (2013)

5. Chen, M., Kong, L., Hong, Y.: Efficient structure-preserving schemes for good Boussinesq equation. Math. Methods Appl. Sci. 41(5), 1743-1752 (2018)

6. Cheng, K., Feng, W., Gottlieb, S., Wang, C.: A Fourier pseudo-spectral method for the 'good' Boussinesq equation with second order temporal accuracy. Numer. Methods Partial Differ. Equ. 31, 202-224 (2014)

7. Chertock, A., Christov, C.I., Kurganov, A.: Central-upwind schemes for the Boussinesq paradigm equation. In: Computational Science and High Performance Computing IV, NNFM, vol. 113, pp. 267-281 (2011)

8. Christou, M., Christov, C.I.: Fourier-Galerkin method for 2D solitons of Boussinesq equation. Math. Comput. Simul. 74, 82-92 (2007)

9. Christov, C.: Conservative difference scheme for Boussinesq model of surface water. In: Proc. ICFD 5, pp. 343-349. Oxford University Press, London (1996)

10. Christov, C.I.: An energy-consistent dispersive shallow-water model. Wave Motion 34, 161-174 (2001)

11. Christov, C.I., Velarde, M.: Inelastic iteration for Boussinesq solutions. Int. J. Bifurc. Chaos 4, 1095-1112 (1994)

12. De Frutos, J., Ortega, T., Sanz-Serna, J.M.: Pseudospectral method for the "good" Boussinesq equation. Math. Comput. 57, 109-122 (1991)

13. Hairer, E., Lubich, C., Wanner, G.: Geometric numerical integration illustrated by the Störmer-Verlet method. Acta Numer. 12, 399-450 (2003)

14. Hairer, E., Lubich, C., Wanner, G.: Geometric Numerical Integration (Structure-Preserving Algorithms for Ordinary Differential Equations). Springer, Berlin (2006)

15. Huang, L., Zeng, W., Qin, M.: A new multisymplectic scheme for nonlinear 'good' Bousinesq equation. J. Comput. Math. 21(6), $703-714$ (2003)

16. Kolkovska, N.: Two families of finite difference schemes for a multidimensional Boussinesq equation. AlP Conf. Proc. $1301,395-403$ (2010)

17. Kolkovska, N.: Convergence of finite difference schemes for a multidimensional Boussinesq equation. In: Numerical Methods and Applications. NMA 2010, LNCS, vol. 6046, pp. 469-476 (2011)

18. Kolkovska, N.: Four level conservative finite difference schemes for Boussinesq paradigm equation. AIP Conf. Proc. $1561,68-74$ (2013)

19. Kolkovska, N., Dimova, M.: A new conservative finite difference scheme for Boussinesq paradigm equation. Cent. Eur. J. Math. 10(3), 1159-1171 (2012)

20. McLachlan, R.: Symplectic integration of Hamiltonian wave equations. Numer. Math. 66, 465-492 (1994)

21. Mehdi, D., Rezvan, S.: A meshless based numerical technique for traveling solitary wave solution of Boussinesq equation. Appl. Math. Model. 36, 1939-1956 (2012)

22. Ortega, T., Sanz-Serna, J.M.: Nonlinear stability and convergence of finite-difference methods for the "good" Boussinesq equation. Numer. Math. 58(2), 215-229 (1990)

23. Pani, A., Saranga, H.: Finite element Galerkin method for the "good" Boussinesq equation. Nonlinear Anal., Theory Methods Appl. 29, 937-956 (1997)

24. Samarsky, A.: The Theory of Difference Schemes. Dekker, New York (2001)

25. Wang, S., Chen, G.: Cauchy problem of the generalized double dispersion equation. Nonlinear Anal., Theory Methods Appl. 64, 159-173 (2006)

26. Yan, J., Zhang, Z.: New energy-preserving schemes using Hamiltonian boundary value and Fourier pseudospectral methods for the numerical solution of the "good" Boussinesq equation. Comput. Phys. Commun. 201, 33-43 (2016)

27. Zhang, C., Huang, J., Wang, C., Yue, X.: On the operator splitting and integral equation preconditioned deferred correction methods for the "good" Boussinesq equation. J. Sci. Comput. 75, 687-712 (2018) 